\title{
EchoGéo
}

19 | 2012

Du littoral à la haute mer : quelles recherches récentes en géographie?

\section{La haute mer : un espace aux frontières de la recherche géographique}

\section{Camille Parrain}

\section{OpenEdition}

\section{Journals}

Édition électronique

URL : https://journals.openedition.org/echogeo/12929

DOI : $10.4000 /$ echogeo.12929

ISSN : 1963-1197

Éditeur

Pôle de recherche pour l'organisation et la diffusion de l'information géographique (CNRS UMR 8586)

Référence électronique

Camille Parrain, «La haute mer : un espace aux frontières de la recherche géographique », EchoGéo

[En ligne], 19 | 2012, mis en ligne le 10 février 2012, consulté le 10 août 2021. URL : http://

journals.openedition.org/echogeo/12929; DOI : https://doi.org/10.4000/echogeo.12929

Ce document a été généré automatiquement le 10 août 2021.

EchoGéo est mis à disposition selon les termes de la licence Creative Commons Attribution - Pas d'Utilisation Commerciale - Pas de Modification 4.0 International (CC BY-NC-ND) 


\title{
La haute mer : un espace aux frontières de la recherche géographique
}

\author{
Camille Parrain
}

\section{Introduction}

1 L'océan a longtemps été perçu comme une frontière et est encore considéré comme tel dans les recherches en géographie. Il est pourtant un lien pour les sociétés et aussi pour la géographie un pont intra et interdisciplinaire. Une des principales difficultés pour analyser l'océan tient à la nature propre de cet espace (liquide) et à son immensité qui lui confèrent une forte altérité. C'est un espace physique complexe qu'il est nécessaire de saisir car prédominant même si on ne peut se vouloir déterministe. Malgré le caractère changeant et mouvant de l'espace maritime une certaine permanence des phénomènes montre la dualité de ce milieu que l'on retrouvera fréquemment. L'océan est également un espace aux multiples ressources et par conséquent aux nombreux enjeux. "L'océan est désormais conçu comme un espace technique, stratégique, économique, juridique et même touristique " (Vanney, 1991, p.11). Il suscite une conquête multidimensionnelle (surface, profondeur). 
Illustration 1 - La mise en place d'un espace multidimensionnel

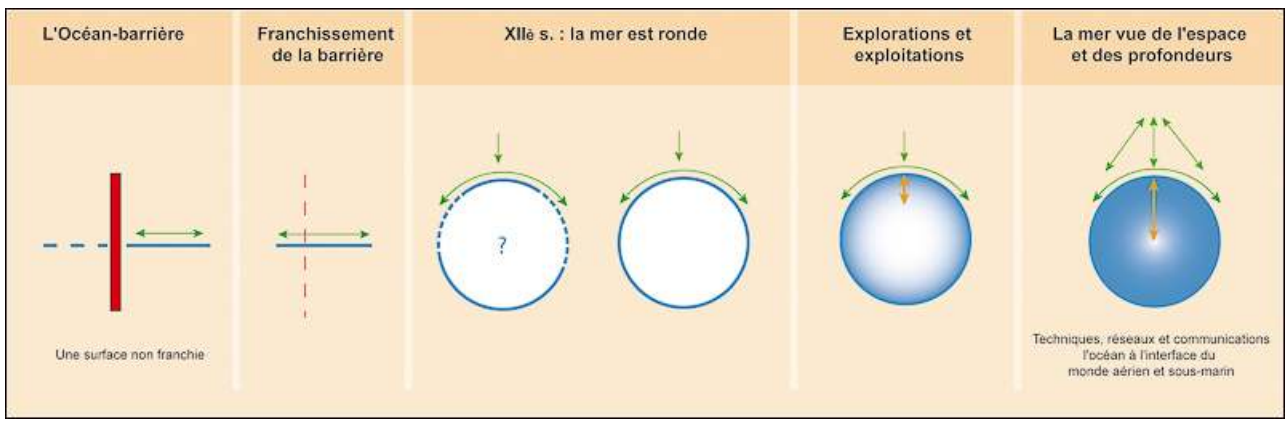

Conception/ Réalisation : C. Parrain, P. Brunello.

2 Cependant, les principales analyses portent sur les zones côtières et au sein de la Zone Economique Exclusive (ZEE) mais, au-delà, les cartographies le représentent souvent comme vide. Les recherches existantes ne placent pas toujours l'océan au centre de la réflexion. Si l'océan a été étudié d'un point de vue physique dans sa globalité, la géographie humaine de l'océan reste encore pionnière.

Après avoir présenté les particularités de la haute mer, nous ferons un état des lieux de la recherche sur les océans et nous nous concentrerons sur des exemples d'analyse basés sur la navigation à voile, les territoires et les paysages.

\section{Partir en mer à l'interface des différentes disciplines}

\section{La particularité de la haute mer}

Qu'est-ce que la haute mer? Pour le droit, elle se définit par exclusion: il s'agit de «toutes les parties de la mer qui ne sont comprises ni dans la zone économique exclusive, la mer territoriale ou les eaux intérieures d'un Etat, ni dans les eaux archipélagiques d'un Etat archipel»(Convention des Nations Unies). Cette définition aura des conséquences dans l'acceptation sociale, la représentation et l'utilisation de cet espace.

Mais la haute mer dépasse la simple définition juridique. Elle fait aussi partie de toute représentation des usagers, qu'il s'agisse des pêcheurs, des marins de la marine marchande, des navigateurs à la voile. Ainsi, par exemple, les navigateurs à la voile se sentent en haute mer avant même d'avoir dépassé la frontière des 200 milles de la ZEE. Leur ressenti repose sur l'absence de la visibilité de la terre, l'état de la mer ou un rapport temporel (être à plus de 24 heures d'une côte, soit avoir passé au moins une nuit en mer). A partir d'enquêtes menées auprès des plaisanciers ${ }^{1}$, la haute mer en général est décrite comme étant homogène car bleue mais ce bleu particulier (bleu profond) l'identifie car introuvable ailleurs. L'océan est aussi cette immensité à laquelle est associée une houle spécifique : une longue houle parfois comparée à des collines ou montagnes selon les zones géographiques. L'océan est aussi variable et vient donc contredire l'aspect monotone et homogène souvent décrit. Il est aussi comparable à d'autres espaces tel le désert et la montagne (nous y reviendrons). C'est un espace d'altérité qui signifie évasion et échappatoire pour les pratiquants de loisir et un espace de vie et parfois de danger pour les professionnels. La citation attribuée à Platon « Il y a 
trois sortes d'hommes: les vivants, les morts et ceux qui vont sur la mer » montrait déjà que l'océan est « autre », un monde à part où il faut oser s'aventurer.

\section{Un terrain difficile}

6 L'océan est un terrain ardu pour le géographe du fait de ses caractéristiques (immense, changeant) mais aussi par sa complexité (liée surtout à l'espace physique et à la nécessaire interdisciplinarité).

7 La principale difficulté pour qui souhaite analyser l'océan consiste à avoir une vision de marin et non de terrien, en se confrontant à l'espace maritime. Le terrien doit se familiariser avec le milieu marin. Il faut donc concevoir de partir et vivre la mer pour l'appréhender. A quelle échelle faut-il se positionner: le long d'itinéraires transatlantiques ou faut-il identifier (au préalable ou après analyse) des zones représentatives au large?

8 Il faut également se soumettre à une temporalité différente : partir en mer nécessite de s'adapter aux saisons (saisons cycloniques, présence de glaces en mer, probabilités de forts coups de vents et de vagues). Le terrain nécessite donc au préalable des connaissances sur les conditions météorologiques prédominantes à un temps $\mathrm{T}$ et, à partir de celles-ci, l'évaluation de sa zone et période de terrain. Le travail de terrain s'insère dans cette temporalité qui nécessite une forte adaptation et disponibilité.

9 Partir faire du terrain en mer demande également d'importants moyens matériels (ne serait-ce qu'une embarcation) pour des enjeux en mer encore sous-estimés. S'il peut être aisé de trouver une embarcation, en revanche, le chercheur devra aussi parfois être navigateur et s'adapter à la vie à bord (quarts, tenir la barre etc.) avec parfois des inconnus sur un espace réduit dans des conditions de navigations imprévisibles. C'est ce qui rend de plus ce terrain intéressant et stimulant car il faut s'immerger. Comme une île, le bateau ressemble parfois à une prison ou à un éden.

10 Surtout, l'étude de l'océan nécessite une réelle interdisciplinarité de recherche, terme en vogue sans réelle méthodologie ni coordination. En ce sens, l'espace maritime permet de la tester et peut être de davantage l'appréhender du fait de la variété des paramètres et de leurs influences sur les usages et usagers. Comprendre l'océan et son humanisation fait également appel à l'océanographie, l'écologie, la climatologie et la météorologie, le droit, l'histoire, l'économie...

11 Avant la mise en place nécessaire de cette interdisciplinarité, la géographie de l'océan a souvent été régionale et sectorielle.

\section{La géographie de l'océan}

\section{Le « mal de l'océan »}

12 «Le mal de l'océan » (Tisser, 1998) au sein de la géographie semble se poursuivre. Les thèmes océaniques reviennent toujours à terre. Les titres des ouvrages : «Le sixième continent » (Papon, 1996), « Laplanète océane » (Louchet, 2009) laissent à entendre que cet espace prend et devrait prendre la même importance que les réflexions terriennes mais ce n'est pas encore le cas. 
$13 \mathrm{Au}$ sein de la géographie française, la géographie de l'océan se trouve en marge. Lorsqu'elle est réalisée, les aspects océaniques sont principalement étudiés sous un regard régional (Vallaux, 1932, 1933 ; Vanney, 1991 ;Louchet, 2009) et l'approche, souvent sectorielle.

14 Ainsi l'aspect physique est mis en avant par Jean-René Vanney (Vanney 1991, 2002), André Guilcher (Guilcher, 1957) et André Louchet (Louchet, 2009). D'autres géographes (Guillaume, 2008; Marcadon, 2001) se sont intéressés aux transports mais l'océan semble passer parfois au second plan, en étant décrit comme un simple support de déplacement, inscrit dans un système réticulaire (Frémont, 2007).

Lessources sont également un champ de recherche important, notamment dans le domaine halieutique (Corlay et Chaussade, 1988). Lorsque le géographe traite de ces ressources en haute mer, l'approche va être principalement locale et régionale en privilégiant une analyse selon les découpages de la FAO ou alors principalement au sein de la ZEE. Des approches sur le comportement des pêcheurs ont toutefois été développées mais principalement sur le côtier.

Enfin les géographes s'intéressent également à l'aspect juridique et géopolitique (Vigarié, 1995 ; Marcadon, 2001 ; Vallega, 2001 ; Coutau-Bégarie, 2007) et sur la manière dont le partage de l'océan se concrétise. La gouvernance représente souvent le principal champ d'analyse de la mer hauturière (Rochette et al., 2008) et regroupe d'ailleurs plus de biologistes, environnementalistes et juristes que de géographes. Plus récemment, les recherches sur les activités humaines en mer, la gestion intégrée de la zone côtière et la planification spatiale (Trouillet 2004, 2006; Trouillet et al., 2011) développent une approche plus globale et systémique mais portent essentiellement sur l'espace côtier et non sur le hauturier même si on assiste à des extensions de projets vers le large comme le montre la convention pour la protection du milieu marin de l'Atlantique du Nord-Est (OSPAR).

17 L'environnement entre sur le devant de la scène et constitue pour la géographie de l'océan une bonne interface au sein à la fois de la géographie elle-même mais aussi entre géographie et autres disciplines. Sur le côtier, on est encore en phase d'acquisition de données (stade avancé) et surtout de coordination de connaissances. Pour le hauturier, l'acquisition de connaissances est encore en cours.

\section{L'océan comme système et enrichissement théorique}

18 Les océans ont été étudiés à partir d'un objet mais non à partir d'un système intégré entre activités humaines et milieu physique (Steinberg, 2001). Pourtant cet espace est un véritable système. Les différentes conceptions de l'espace qu'elles soient représentatives, physiques ou géopolitiques sont liées. C'est un système de fonctionnement interne (l'océan) et de double fonctionnement externe-interne (les différents usagers et les cinq océans).

Les représentations de la mer restent encore trop terriennes et n'insistent pas assez sur le mouvement. De nouvelles approches et cartographies commencent à redessiner l'espace maritime en fonction des activités humaines mais principalement sur la mer côtière (Brigand et al., 2003, 2006; Le Berre et al,. 2010 ; Le Tixerand et Gourmelon, 2009 ; Gray et al., 2011) ou ne sont pas toujours réalisées par des géographes (Halpern et al., 2008 ; Breeze et al., 2005). 

regroupant différents sous-groupes (croisière, régate, convoyage, navigation en solitaire ou en équipage). Celle-ci était intéressante du point de vue de la proximité entre navigateurs et océans, principalement liée au mode de propulsion (la voile). Il s'agira désormais d'étendre cette analyse à d'autres usages afin de comparer les différentes constructions territoriales et de les combiner.

Cependant, dans le domaine de la géographie humaine des océans, beaucoup de pistes restent à approfondir : analyser les relations usagers-océan, recouper les informations afin de mener des synthèses sur les phénomènes de socialisation et humanisation des océans car "L'océan n'est pas simplement un espace utilisé par la société; il est un composant de l'espace des sociétés »(Steinberg, 2001, p. 20).

Les recherches principalement appliquées doivent être complétées par des recherches théoriques encore quasiment absentes en ce qui concerne l'espace hauturier. L'océan permettrait également de transposer en mer certains concepts développés à terre afin de les réadapter et les redéfinir. Ils pourraient aussi être réintégrés à terre (mobilité, territoire, réseaux, paysage, frontières).

Des rapprochements peuvent être faits avec la montagne et les déserts. Ces deux espaces constituent également des extrêmes et des «territoires du vide ». La montagne se rapproche de la mer par ses pratiques de loisir des sociétés (quête de l'aventure, dépassement de soi, isolement) et le désert par son apparente homogénéité et la difficulté d'y trouver des repères. La montagne diffère de ces deux milieux du fait de sa « rigidité », de ses repères fixes et de son hétérogénéité.

Comme dit précédemment, l'interdisciplinarité est une clé pour mieux appréhender cet espace et il conviendrait de davantage intégrer le physique et l'humain plutôt que de procéder à une segmentation. Dans le domaine de la gestion des ressources vivantes les modèles intègrent de plus en plus le paramètre humain sous l'angle géographique, économique et anthropologique (tactique, stratégie, comportements) et biologique (structure des espèces, migrations) (Guénette et al., 2007). Il est primordial de s'ouvrir sans pour autant oublier sa propre identité de géographe.

Une ouverture intéressante est celle de la "géographie écologique des océans » d'Alan Longhurst (Longhurst, 2007) qui dresse un découpage océanique à partir de paramètres «biogéographiques ». Il conviendrait d'élargir cet angle en intégrant davantage l'humanisation des océans.

\section{Essai sur les territoires de la navigation à voile}

La principale difficulté réside dans la compréhension de cet espace vaste, liquide, peu connu, reflétant l'altérité. Un des principaux enjeux est d'arriver à se déraciner de la terre (sans l'oublier) et de créer de nouvelles cartographies insistant sur la particularité de l'espace maritime. Le but est de comprendre la structuration de l'océan et de coupler les interactions homme-océan afin de permettre une meilleure gestion et protection en lien avec les recherches menées dans d'autres disciplines. Nous avons donc affiné les concepts de territoire et de paysage en mer qui sont primordiaux pour démontrer les enjeux environnementaux et juridiques et peuvent même être des outils de gestion. 
Illustration 2 - Complexité des relations usagers et océans : exemple de l'analyse de la navigation à voile

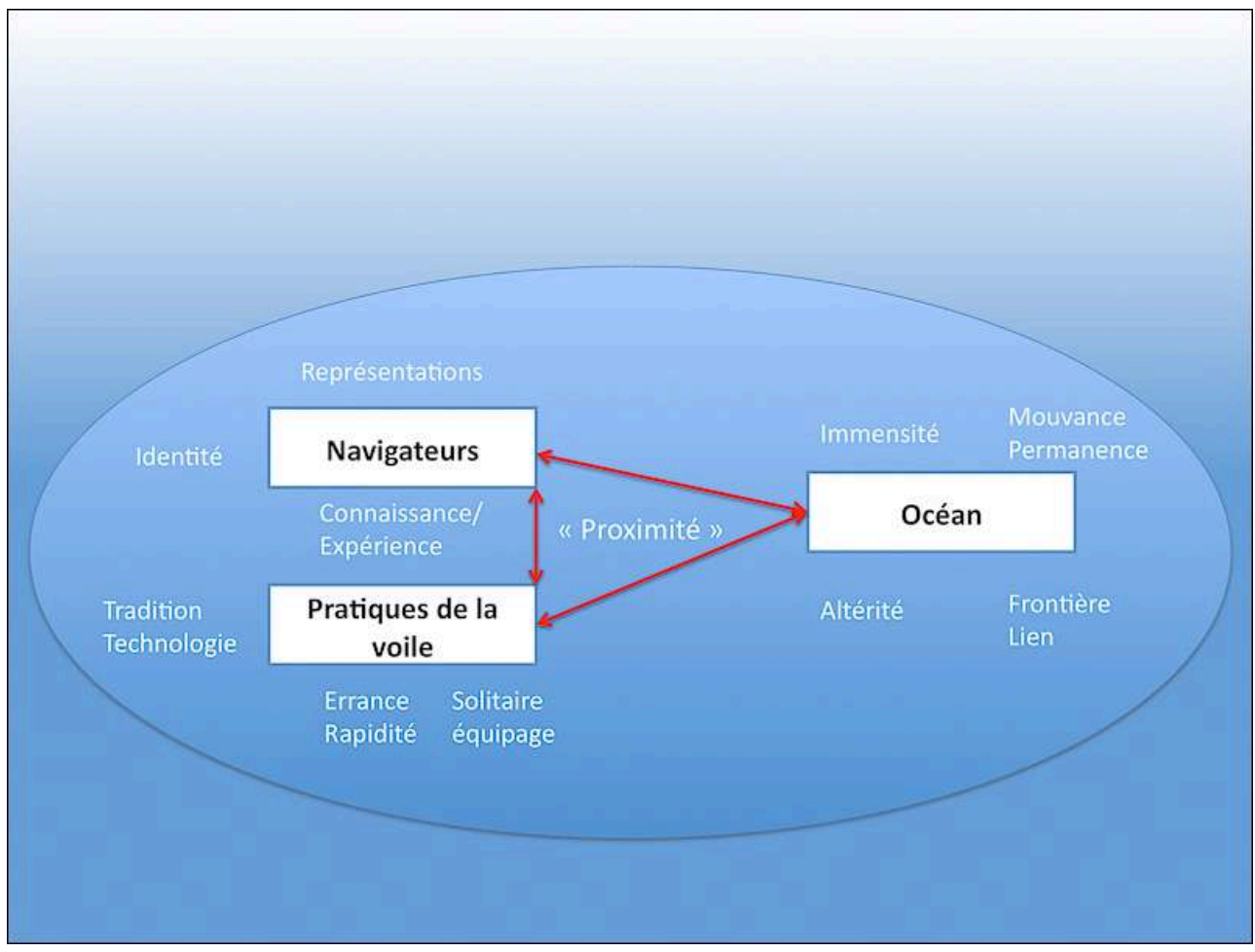

Conception/Réalisation : C. Parrain

\section{Du territoire au « merritoire » : mobilité, discontinuités et réseaux}

Le territoire en mer, que nous qualifierons de «merritoire » a été la principale entrée de réflexion. Nous employons le terme de merritoire car le concept de territoire ne peut avoir complètement le même sens qu'à terre du fait des caractéristiques particulières de l'océan dont la principale est l'hypermobilité (espace mouvant et hommes en déplacement). Il se rapprocherait donc d'un territoire éphémère. Pour l'évaluer, on s'est basé sur les itinéraires transatlantiques. Nous avons donc analysé premièrement l'océan selon une structuration linéaire. Les hommes y ont des repères et créent leurs systèmes de représentations (révélés par enquêtes et récits) qui, complétés par des observations in situ le long de ces itinéraires, permettent de déceler des aires atlantiques homogènes (houle, couleur de l'eau, nuages, faune, algues, trafic, déchets, systèmes de représentation) et des discontinuités marquées par des rites ou des discours communs (structuration aréale). Il conviendrait de compléter les informations sur les parcours des usagers (autre pratique, autre bassin de navigation), sur les éléments observés en mer ainsi que sur l'analyse de leurs discours (récits, vacations radios des courses au large) afin de développer le corpus cartographique en mer. Les discontinuités révélées (rupture paysagère, modifications des comportements des usagers) pourraient constituer des délimitations dynamiques. L'organisation de l'océan est aussi construite à partir de réseaux de communication et sociaux. Comment ces réseaux contribuent-ils à connecter ou déconnecter les navigateurs entre eux et avec l'océan ? En quoi l'amélioration des moyens de communication (internet à bord, réception des bulletins météorologiques, téléphone satellite) permet-elle ou non de se 
concentrer sur l'espace maritime, d'obtenir de nouveaux regards? Si le marin peut être connecté à terre, se déconnectera-t-il de la meret en fera-t-il un simple lieu de passage ? Ou est-ce que les échanges en mer permettront de s'affranchir de certaines difficultés et dès lors de se concentrer différemment sur l'océan?

L'ensemble de ces paramètres permet d'obtenir une combinaison de structurations océaniques (illustration 3). Nous allons nous attacher à une des formes d'évaluation du territoire : le paysage.

Illustration 3 - Structuration des territoires en haute mer

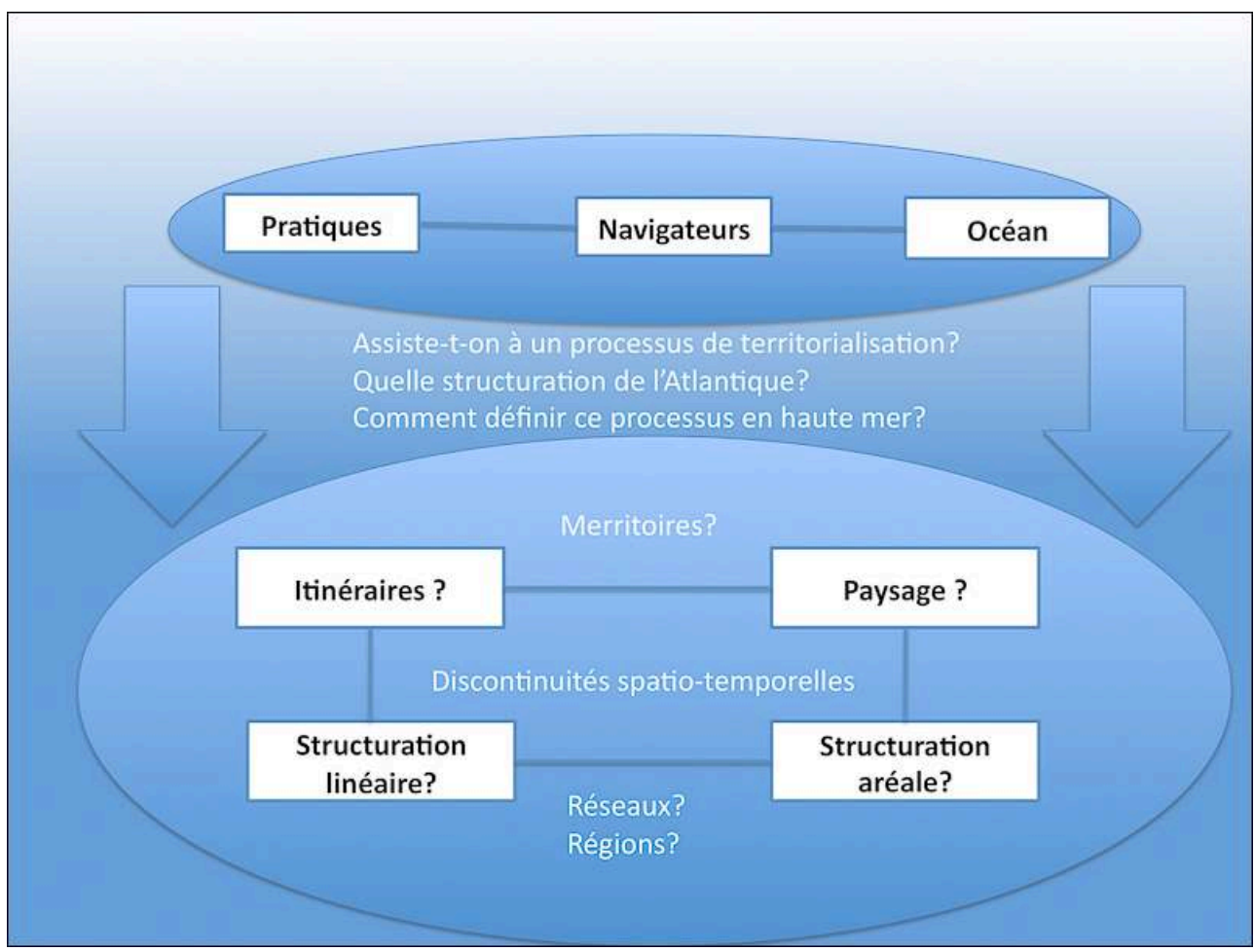

Conception/Réalisation : C. Parrain.

\section{Paysage marin, environnement et gestion}

L'apport du géographe dans l'approche paysagère enrichit le concept de paysage appliqué à l'espace maritime. Pour l'élaboration d'aires marines protégées, le paysage est principalement analysé sous l'angle des biologistes et concerne donc les habitats (référence à la bathymétrie). Si un séminaire de l'Agence des Aires Marines Protégées en 2011 a montré l'intégration progressive de la participation des plongeurs dans la définition du paysage sous-marin, elle reste encore faible. Les paysages marins ont aussi leur place dans l'évaluation d'aires marines protégées. Ils sont à l'interface pratiquants-éléments observables et sensibles et prennent la forme de paysageitinérant. Ils constituent des repères et références pour les hommes et fournissent également des informations sur les éléments présents en mer. De ce fait, le paysage constitue à notre sens un outil de gestion pour l'espace maritime; il est primordial pour arriver à délimiter et faire accepter des zones de protection.

Ce travail nécessite un approfondissement des connaissances physiques dans le domaine d'autres disciplines (données océanographiques et biologiques). Il est 
nécessaire de se familiariser avec les aspects faunistiques (aires de répartition, migration et stratégies alimentaires de l'avifaune, des mammifères marins, des poissons et méduses) et avec les aspects biogéographiques (plancton, algues) mais aussi climatologiques et météorologiques (température, salinité, systèmes de front, types de nuages, houle associée etc.). À titre d'exemple, des observations in situ ont consisté à relever trois fois par jour et ce à la même heure les conditions météorologiques mais aussi toute présence de faune, algues, pollution et déchets en mer et à mentionner toute communication réalisée en mer avec un autre navire en précisant le nom du navire, la provenance, la destination etc. L'analyse paysagère ne doit pas se contenter d'un travail d'observation mais doit aussi être complétée par l'analyse de récits et de discours (illustration 4).

Illustration 4 - L'analyse paysagère

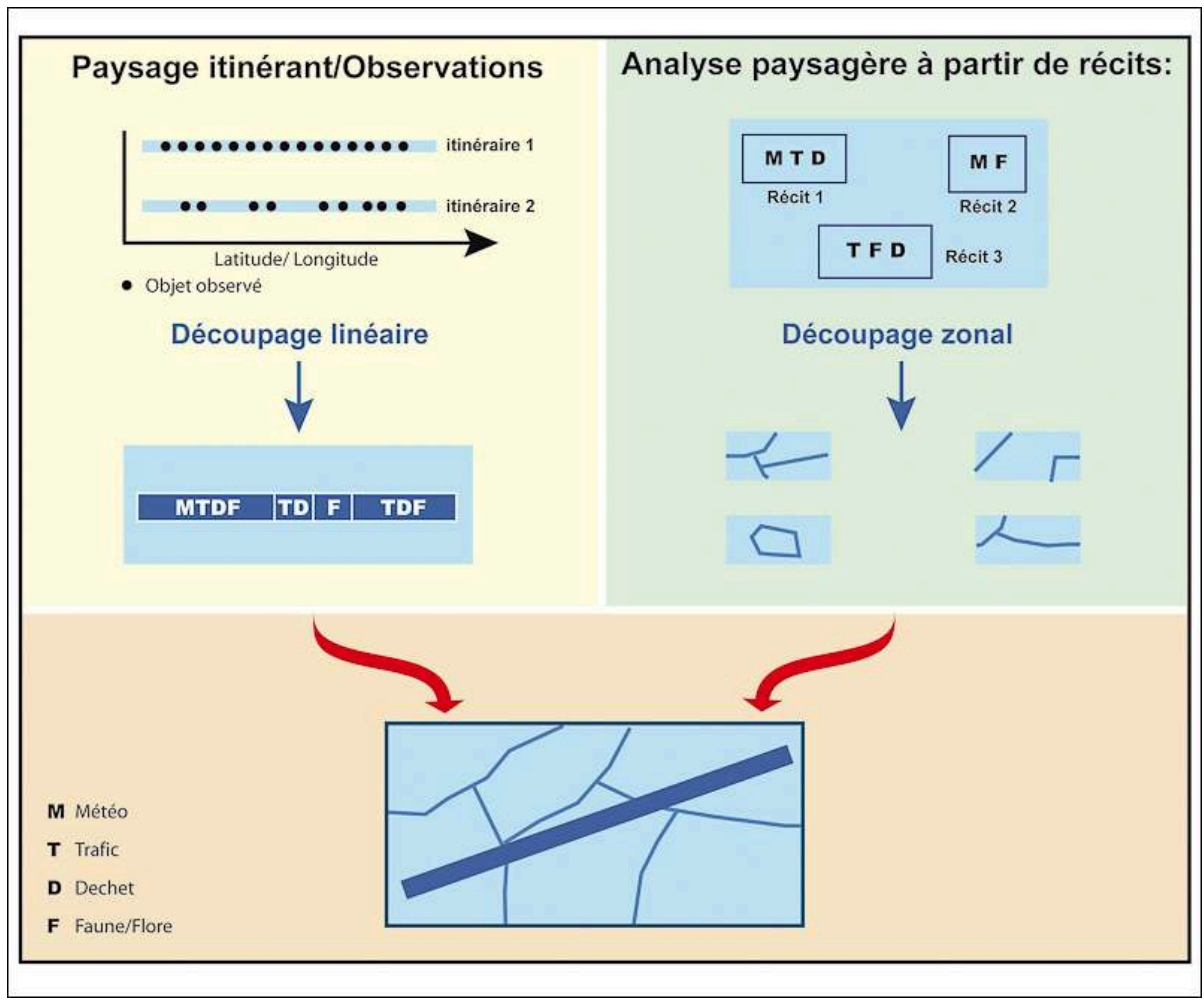

Conception/Réalisation : C. Parrain, P. Brunello.

\section{Conclusion}

31 La haute mer est un champ d'étude encore à explorer. Cet espace connaît différents enjeux sur le plan de la géopolitique, des ressources et de l'environnement. Les aires marines protégées s'étendent par exemple de plus en plus vers le large et il est primordial d'intégrer les activités humaines et la relation homme-océan pour toute élaboration de zone protégée. Gestion et planification spatiale représentent des champs de recherche en devenir. Des connaissances et observations sont nécessaires dans un premier temps (recherche appliquée) puis une orientation conceptuelle serait intéressante pour souligner la particularité de cet espace et pourrait aider à comprendre d'autres milieux et phénomènes sur les continents. Ainsi, à titre d'exemple 
avec la mobilité accrue des sociétés, le paysage se déroule le long d'itinéraires précis et multiples.

La compilation et l'analyse des informations présentées devraient donc permettre l'évaluation d'une zonation en mer, grâce aux liens avec les autres disciplines, et la transposition des concepts à terre (territoire de la mobilité, paysage en mouvement) et à d'autres milieux (désert, montagne). Si des événements sportifs réunissent marins et montagnards et si certains se reconvertissent pour l'autre milieu, cela prouve aussi qu'il existe des similitudes entre les pratiques.

L'océan est un espace de plus en plus convoité qu'il convient d'étudier davantage afin d'obtenir de nouveaux entendements car comme le dit Jules Michelet «c'est par la mer qu'il convient de commencer toute géographie ».

\section{BIBLIOGRAPHIE}

Breeze et al, 2005. The Scotian Shelf: An Atlas of Human Activities. Oceans and Coastal Management DivisionOceans and Habitat BranchFisheries and Oceans Canada, Maritimes RegionP.O. Box 1006, http://intra.ent.dfo-mpo.ca/e0009630

Brigand L., Peuziat, I., 2003. Etude de la fréquentation nautique de plaisance dans les archipels de Glénan, Molène et Bréhat. Etat des lieux et perspectives pour la gestion. Programme Life "Archipels et îlots marins de Bretagne", Géomer, UMR 6554 LETG, Université de Bretagne Occidentale, Association pour la Promotion et la Protection des Îles du Ponant, 84 p.

Chaussade J., Corlay, J., 1988. Atlas des pêches et des cultures marines en France. Ed. GIP Reclus, 104 p.

Frémont A., 2007. Global maritime networks. The case of Maersk. Journal of Transport Geography, vol. 15, n 6, p. 431-442 (base ISI).

Gray L. et al., 2011. Spatial characterization of marine recreational boating: Exploring the use of an on-the-water questionnaire for a case study in the Pacific Northwest. Marine Policy, Volume 35, Issue 3, p 286-298.

Guénette S. et al., 2007. A synthesis of research activities at the Fisheries Centre in ecosystembased fisheries modelling and assessment with emphasis on the Northern and Central Coast of BC. Fisheries Centre Research Reports, Vancouver, Volume 15, number 1, 32 p.

Guilcher A., 1957. Océanographie (hydrologie des océans et des mers). Paris, CDU, 191 p.

Guillaume J., 2008. Les transports maritimes dans la mondialisation. Paris, L'Harmattan, 275 p.

Halpern B.S. et al., 2008. A global map on human impact on marine ecosystems. Science, vol. 319. $\mathrm{n}^{\circ} .5865, \mathrm{p} .948-952$.

Le Berre S., 2008. Les observatoires de la fréquentation, outils d'aide à la gestion des îles et des littoraux. Vol. 1 L'envers du décor. Vol. 2 Les éléments du décor. Thèse de doctorat en géographie, Géomer LETG UMR 6554, Université de Bretagne Occidentale, 745 p. 
Le Berre S. et al., 2010. Etude de la fréquentation nautique du Bassin d'Arcachon. Rapport laboratoire Géomer UMR 6554 LETG, Université de Bretagne Occidentale, Direction départementale des Affaires maritimes de Gironde, $94 \mathrm{p}$.

Le Tixerand M., Gourmelon F., 2009. Approche dynamique du déroulement d'activités humaines en mer côtière. Cybergeo : European Journal of Geography, Systèmes, Modélisation, Géostatistiques, article 333, mis en ligne le 06 janvier 2006, modifié le 26 juin 2009. http://cybergeo.revues.org/ 2938

Longhurst A., 2007. The ecological geography of the sea. Burlington, Ed. Academic Press, 542 p. Louchet A., 2009. La planète océane : précis de géographie maritime. Paris, Ed. A. Colin, 559 p.

Marcadon J., 2001. Les enjeux atlantiques. Paris, Ed. Ellipses, 157 p.

Michelet J., 1983. La mer. Paris, Ed. Gallimard, 1 ère édition 1861, 409 p.

Papon P., 1996. Le sixième continent : géopolitique des océans. Paris, Ed. O. Jacob, 336 p.

Rochette J. et al., 2008. Towards a new governance of High Seas biodiversity. Oceanis, $\mathrm{n}^{\circ} 35,292 \mathrm{p}$. Steinberg P.E., 2001. The social construction of the ocean. Cambridge, Ed. Cambridge University Press, $239 \mathrm{p}$.

Tissier J.-L., 1998. L’Atlantique un regard géographique. In Marrou L., Miossec A. (sous dir.), Historiens et géographes, $n^{\circ} 363$, p. 66-312

Trouillet B., 2004. La « mer côtière » d'Iroise à Finisterre. Étude géographique d'ensembles territoriaux en construction. Nantes, Université, thèse de doctorat, $293 \mathrm{p}$.

Trouillet B., 2006. La mer-territoire ou la banalisation de l'espace marin (golfe de Gascogne). M@ppemonde, $\mathrm{n}^{\circ} 84$ (4-2006), Montpellier, 17 p.

Vallaux C., 1932. Mers et océans. Paris, Ed. Rieder, 100 p.

Vallaux C., 1933. Géographie générale des mers. Paris, Ed. Félix Alcan, 796 p.

Vallega A., 2001. Ocean governance in post-modern society-a geographical perspective. Marine Policy, n² 25, p. 399-414.

Vallega A., 2001. Sustainable Ocean governance. London, New York, Routledge, 274 p.

Vanney J-R., 1991. Introduction à la géographie de l'océan : une vue nouvelle du monde. Paris, Ed. Institut Océanographique, $214 \mathrm{p}$.

Vanney J.-R., 2002. Géographie de l'océan Global. Paris, Ed. scientifiques GB, 335 p.

Vigarié A., 1995. La mer et la géostratégie des nations. Paris, Ed. Economica, Institut de stratégie comparée, $432 \mathrm{p}$.

\section{NOTES}

1. Les enquêtes ont été menées en juin 2008 aux Açores. Une partie de ces enquêtes traitait des représentations maritimes et de la haute mer en général, c'est ce qui est présenté ici. 


\section{RÉSUMÉS}

La haute mer est un espace encore peu étudié au sein de la géographie. Ses caractéristiques en font un espace complexe et reflètent son altérité. L'océan est un terrain difficile et original permettant de se confronter à l'interdisciplinarité (géographie, océanographie, climatologie, biologie, histoire, économie). Les approches menées au sein de la géographie sont souvent régionales, sectorielles, côtières et appliquées et peu cherchent à s'orienter vers des approches théoriques ou vers la haute mer. Nous ferons ici un état des lieux de la place de l'océan au sein de la géographie et tâcherons d'ouvrir des perspectives de recherche en prenant l'exemple de la navigation à voile et de la notion de territoire en haute mer que nous qualifierons de «merritoire». Pour le définir, nous proposons d'ouvrir sur les notions de mobilité, discontinuités, réseaux et de paysage marin. Le paysage, reflet de l'interdisciplinarité, apparaît également comme étant une piste pour la gestion de l'espace maritime.

The high sea has been little studied within geography. Its characteristics show that it is a complex space and do reflect its alterity. The ocean is a difficult and original fieldwork that invites to be analyzed with interdisciplinary methods (geography, oceanography, climatology, biology, history, economy). The geographical approach has so far mainly been an applied geography and has focused on regional, sectorial and coastal aspects. Few are aiming at theoretical approaches or on the high seas. We will overview the position of the ocean within geography and will try to explore new research perspectives by taking into account sailing and the concept of territory in the high sea that we will define as "maritory ». To define it, we offer to discuss on the terms of mobility, discontinuity, network and seascape. Seascape - example of an interdisciplinary tool- could appear as a method for marine management.

\section{INDEX}

Mots-clés : état des lieux, haute mer, interdisciplinarité, paysage, territoire

Keywords : high sea, interdisciplinarity, landscape, overview, territory

Thèmes : Sur le Champ - Sur le Terrain

\section{AUTEUR}

\section{CAMILLE PARRAIN}

Camille Parrain, camille.parrain@univ-nantes.fr, est Post-doctorante, Université de Nantes, LETG-Géolittomer-UMR 6554. Elle a publié :

- Parrain C., à paraître. Territorialisation des espaces océaniques hauturiers: l'apport de la navigation à voile dans l'Océan Atlantique. Editions PUR.

- Parrain C., 2009. Le Vendée Globe ou l' «Everest des mers » :comment revisiter le « Sixième Continent? In les Cafés Géographiques, Vox Geographi, http://www.cafe-geo.net/article.php3?id_article=1509 - Parrain C., 2007. La Route du Rhum : regards sur l'Atlantique de St Malo à Pointe-à- Pitre. 132ème Congrès national des Sociétés Historiques et Scientifiques, «Images et Imageries », Arles 16-23 avril 2007. 